\title{
Prix Prud'homme 2011 \\ Quantifier I'utilisation du dihydrogène par les sols : de l'échelle locale à l'échelle globale
}

Camille Yver

Scripps Institution of Oceanography, University of California, San Diego Gilman Drive, San Diego, CA, 92193

cyver@ucsd.edu

\section{Résumé}

L'utilisation du dihydrogène $\left(\mathrm{H}_{2}\right)$ par les sols est un phénomène encore mal connu qui représente cependant le terme le plus important du bilan de $\mathrm{H}_{2}$. Afin de réduire les incertitudes liées à son dépôt dans le sol, des études à l'échelle locale permettent de calculer la vitesse de ce dépôt. Ces études incluent la mesure de la concentration en $\mathrm{H}_{2}$ mais aussi celle du monoxyde de carbone et du radon. L'utilisation de la modélisation inverse permet de passer de l'échelle locale à l'échelle globale.

\section{Abstract}

Estimating molecular hydrogen soil uptake: from local to global scale

Molecular hydrogen deposition is a phenomenon which is still not well understood. It is, however, the largest term in the $\mathrm{H}_{2}$ budget. The uncertainties in $\mathrm{H}_{2}$ deposition can be reduced by local studies measuring the concentrations of $\mathrm{H}_{2}$, carbon monoxide and radon which allow the rate of the uptake to be calculated. An estimate of this term on a global scale can be made using inverse modelling.

\section{Pourquoi s'intéresser au dihydrogène?}

À cette question, on peut apporter plusieurs réponses.

D'abord, la raréfaction progressive des ressources fossiles ainsi que la nécessité de réduire les émissions de gaz à effet de serre créent un besoin de nouvelles sources d'énergie. Dans ce contexte, le dihydrogène $\left(\mathrm{H}_{2}\right)$ est assuré de jouer un rôle important à travers le développement des piles à combustible.

De plus, bien que le $\mathrm{H}_{2}$ n'influence pas directement le bilan radiatif de l'atmosphère, il affecte la capacité oxydante de celle-ci, via sa réaction avec le radical hydroxyle $(\mathrm{OH})$. Des niveaux plus élevés de dihydrogène entraîneraient une augmentation de la durée de vie de beaucoup de composés, notamment du méthane, ce qui fait de $\mathrm{H}_{2}$ un gaz à effet de serre secondaire.

Des modifications de la concentration de $\mathrm{H}_{2}$ dans la troposphère affectent aussi la quantité de vapeur d'eau dans la stratosphère. Cela influence le bilan radiatif (plus de vapeur d'eau tend, par rayonnement, à refroidir la stratosphère) et joue également un rôle dans la chimie des zones polaires qui concourt au trou de la couche d'ozone.

Bien que cela soit peu probable à l'heure actuelle, on peut concevoir que le changement climatique pourrait entraîner des perturbations dans la chimie et la physique des sols qui ont une influence majeure dans le cycle de $\mathrm{H}_{2}$. Ce phénomène pourrait conduire à une modification de sa concentration atmosphérique.
Enfin, une meilleure connaissance du cycle de $\mathrm{H}_{2}$ aiderait à comprendre le cycle d'autres gaz à effet de serre tel le monoxyde de carbone qui a un impact à la fois environnemental et sanitaire.

\section{Qu'avons-nous compris sur le cycle du dihydrogène en 40 ans ?}

Levy (1971) identifie un premier mécanisme de destruction du dihydrogène (aussi appelé puits) en l'incluant dans le cycle du radical $\mathrm{OH}$. Un second puits, plus important est identifié par Schmidt (1974) trois ans plus tard : le dépôt du dihydrogène dans les sols. Durant le même temps, on s'intéresse bien évidemment aux sources de dihydrogène.

Résumons l'état de nos connaissances sur le cycle du dihydrogène (figure 1). Il existe deux puits dans la troposphère : la réaction avec le radical $\mathrm{OH}$ (environ $25 \%$ ) et le dépôt dans les sols (environ $75 \%$ ). Selon Ehhalt et Rohrer (2009) le flux de $\mathrm{H}_{2}$ de la haute troposphère vers la basse stratosphère est quasi nul, étant donné le faible gradient vertical de concentration qui existe entre elles.

Le dihydrogène provient de quatre sources principales qui représentent $90 \%$ de celles identifiées. Ce sont l'oxydation du méthane et des autres hydrocarbures dans la troposphère, qui représente la moitié des sources, la combustion des énergies fossiles (principalement liée au transport automobile, soit environ $20 \%$ ) et la combustion de la biomasse (environ $20 \%$ ). Les $10 \%$ restants sont 


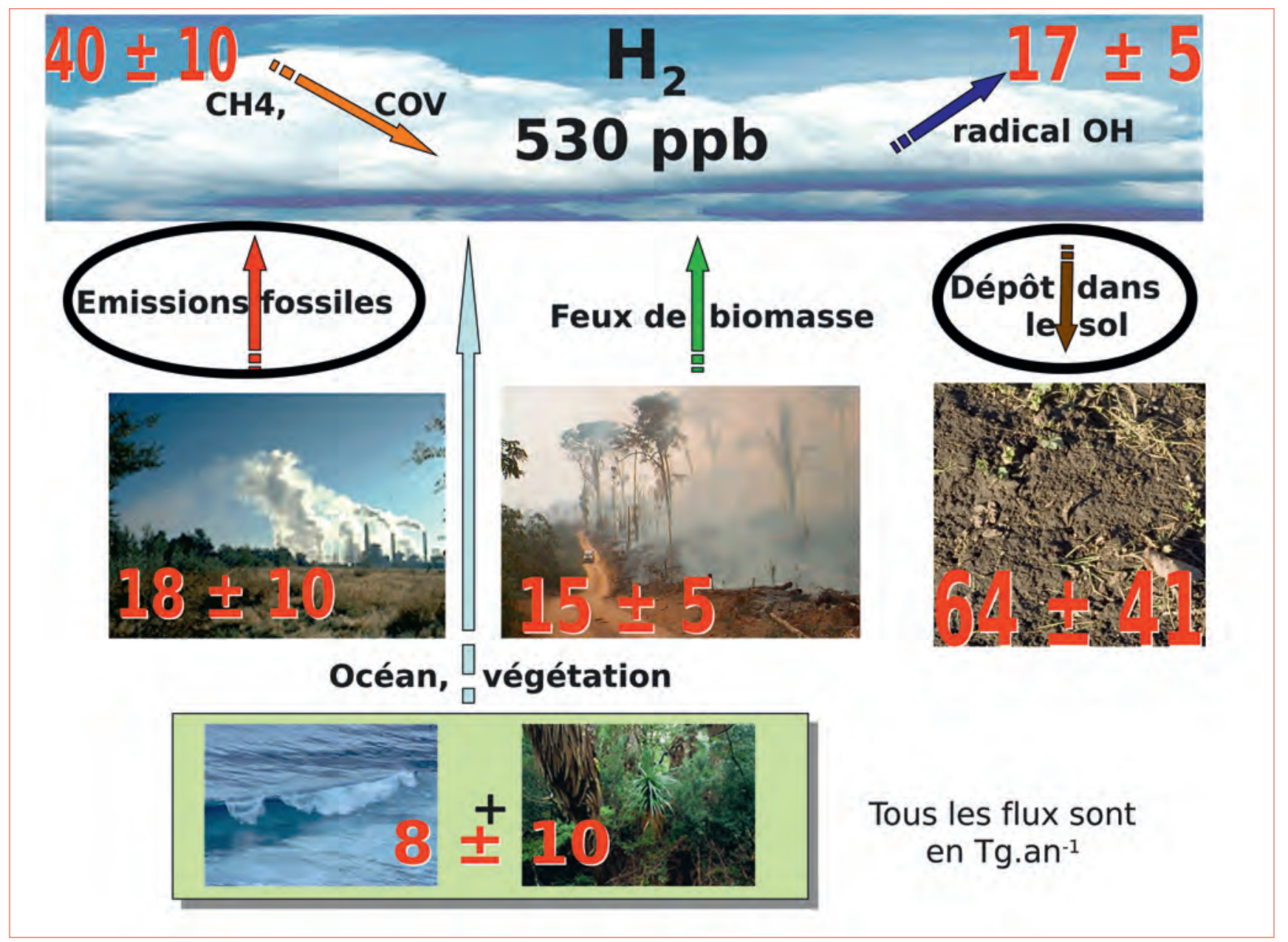

Figure 1 - Représentation schématique du bilan de $\mathrm{H}_{2}$. Les flux sont en $\mathrm{Tg}_{\mathrm{an}}{ }^{-1}$.

principalement dus aux émissions lors de la fixation de l'azote par les végétaux terrestres et marins mais aussi, de façon beaucoup plus marginale (moins de $1 \%$ des sources), aux émissions des océans et des volcans ou à la fermentation.

\section{Le dépôt du dihydrogène : que se passe-t-il dans les dix premiers centimètres d'un sol?}

Le dépôt du dihydrogène dans le sol se fait grâce à l'activité enzymatique d'hydrogénases ou de micro-organismes qui l'utilisent dans leur métabolisme énergétique. Conrad et Seiler (1981) ont montré que ces enzymes étaient responsables de ce dépôt. Par la suite, Conrad et Seiler (1985) et Yonemura et al. (1999, 2000a et b) ont démontré que l'humidité était un facteur clé contrôlant le dépôt de $\mathrm{H}_{2}$. Trop ou trop peu d'humidité inhibe le dépôt. Un compactage faible, des températures plus élevées et une part plus importante de carbone organique sont d'autres facteurs qui entraînent une augmentation de la vitesse de dépôt de $\mathrm{H}_{2}$ (calculée en $\mathrm{cm} \mathrm{s}^{-1}$ ). En effet, ce processus est dépendant de la diffusion pour laquelle les facteurs cités précédemment jouent également un rôle important. Enfin, ces auteurs ont montré que ce processus se jouait dans les dix premiers centimètres du sol. D'abord, $\mathrm{H}_{2}$ diffuse à travers une couche de sol inactive, puis à travers une couche microbiologiquement active. Smith-Downey et al. (2006) ont mené une série d'expériences pour mieux quantifier l'influence de l'humidité et de la température. Ils ont trouvé que le dépôt se produisait même pour des basses températures (moins de $-4{ }^{\circ} \mathrm{C}$ ) et atteignait un optimum à environ $20-30{ }^{\circ} \mathrm{C}$. À $20 \%$ de degré de saturation, le dépôt de $\mathrm{H}_{2}$ est maximum mais il est inhibé pour des degrés plus élevés. S'il y a trop peu d'humidité, les bactéries restent inactives mais, s'il y en a trop, les pores du sol sont remplis et la diffusion ne se fait plus. La première extraction de ces hydrogénases relevée dans la littérature a été menée par Guo et Conrad (2008). Un second article a été publié la même année par Constant et al. (2008). Ces derniers auteurs ont isolé un micro-organisme Streptomyces (PCB7) qui présente une forte affinité avec $\mathrm{H}_{2}$. Cependant, des recherches complémentaires sont nécessaires pour déterminer la nature exacte des enzymes et des micro-organismes intervenant dans l'utilisation du dihydrogène.

On conçoit aisément que tous les paramètres évoqués précédemment entraînent une répartition spatiale et temporelle du dépôt avec un maximum aux saisons chaudes et sèches. En effet, un temps chaud et sec favorise l'activité bactérienne alors qu'en période humide, cette activité est réduite. Dans l'hémisphère nord, on observe un dépôt maximum en été et en automne, et un dépôt minimum en hiver. Dans l'hémisphère sud, le dépôt est très inférieur en raison de la faible superficie des terres émergées.

Le dépôt de $\mathrm{H}_{2}$ dans le sol est un phénomène physique encore mal connu et donc quantifié avec une très grande marge d'erreur. Comme le montre la figure 1 , c'est le terme du bilan de $\mathrm{H}_{2}$ qui comporte le plus d'incertitudes. C'est pourquoi, au Laboratoire des 
sciences du climat et de l'environnement (LSCE), nous avons voulu mieux estimer ce terme afin de réduire les incertitudes sur le bilan du dihydrogène.

Dans la suite de l'article, nous présentons d'abord une étude à l'échelle locale, menée en région parisienne et en région Centre (Yver et al., 2009, 2011a). Ensuite, l'étude est étendue à la planète grâce à l'utilisation de la modélisation (Yver et al., 2011b).

\section{Estimation du dépôt de $\mathrm{H}_{2}$}

\section{Une étude locale sur le dépôt du dihydrogène}

Cette étude a été menée sur deux sites de mesure. Le premier est situé à Gifsur-Yvette, dans l'Essonne, à environ $20 \mathrm{~km}$ de Paris. Le second se trouve sur la commune de Trainou, près de la ville d'Orléans, dans le Loiret. Sur le site de Trainou, les mesures sont effectuées à trois hauteurs, le long d'une tour. Sur les deux sites, on mesure la concentration de $\mathrm{H}_{2}$ ainsi que celles du monoxyde de carbone (CO) et du radon $\left({ }^{222} \mathrm{Rn}\right)$.

En quoi la mesure des concentrations du monoxyde de carbone et du radon présente-t-elle un intérêt pour notre étude?

La mesure des concentrations de monoxyde de carbone est utile car il partage les mêmes sources et les mêmes puits que le dihydrogène, à l'exception du dépôt au sol. Cela nous permet donc de séparer les périodes où le dépôt est le phénomène prédominant de celles où l'influence des autres termes n'est pas négligeable.

Quant au radon, on sait que le ${ }^{222} \mathrm{Rn}$ est émis de façon relativement constante et homogène pour une région donnée. Les variations de sa concentration dans l'atmosphère sont essentiellement dues au transport atmosphérique. Cela en fait donc un bon traceur des masses d'air. Si l'on considère que le dépôt de $\mathrm{H}_{2}$ et l'émission de ${ }^{222} \mathrm{Rn}$ sont des flux constants et colocalisés, qui se produisent dans une couche d'air bien mélangée de hauteur constante, alors la variation au cours du temps des concentrations de ${ }^{222} \mathrm{Rn}$ et $\mathrm{H}_{2}$ nous permet d'estimer le flux de $\mathrm{H}_{2}$ en connaissant celui de $222 \mathrm{Rn}$.

\section{Les instruments utilisés}

Les concentrations de $\mathrm{H}_{2}$ et $\mathrm{CO}$ sont mesurées par chromatographie en phase gazeuse. Cette méthode consiste à séparer les différents composants d'un échantillon, ici l'air prélevé. La séparation se fait le long d'une colonne selon des principes physiques (taille des particules) ou chimiques (affinité des molécules pour le matériel de la colonne). Un gaz vecteur, aussi appelé phase gaz, sert à pousser l'échantillon qui va être plus ou moins retenu par la phase dite stationnaire, constituée par la colonne.

Le temps de parcours de l'échantillon dans la colonne, appelé temps de rétention, est connu pour chaque gaz selon le débit de la phase gaz et la nature de la phase stationnaire. $\mathrm{H}_{2}$ et $\mathrm{CO}$ ont des propriétés physiques différentes (leur taille notamment). Ainsi $\mathrm{H}_{2}$ parcourt-il la colonne plus rapidement que $\mathrm{CO}$ et il est donc analysé en premier.

Chaque gaz arrive ensuite dans la chambre de détection. Le détecteur utilisé est dit détecteur de gaz réduit. En effet, il permet d'analyser tous les gaz qui réduisent l'oxyde de mercure $\mathrm{HgO}$ en vapeur de mercure. C'est la quantité de mercure formée sous forme de vapeur qui est ensuite mesurée par absorption dans l'ultraviolet. Pour déterminer les concentrations de chacun des gaz des échantillons, il est ensuite nécessaire de connaître le coefficient de proportionnalité entre la hauteur du signal et la concentration recherchée. Pour cela, des bouteilles de gaz de concentrations connues sont régulièrement analysées. Avec ce système, la concentration de $\mathrm{H}_{2}$ et $\mathrm{CO}$ est connue avec précision et avec une relativement haute fréquence (deux échantillons par heure, au minimum).

Le radon-222 est un gaz descendant radioactif de l'uranium-238 et du thorium-232. Lors de la désintégration du radium, le radon-222 est émis par les sols et il est donc plus abondant audessus des continents que des océans. Son taux d'émission dépend essentiellement du type de sol et des conditions météorologiques telles que la pression ou les précipitations (Schery et al., 1984). Il varie entre 0 et quelques dizaines d'atomes par centimètre carré et par seconde. Sa demi-vie de 3,8 jours en fait un bon traceur atmosphérique à l'échelle régionale.

Depuis janvier 2002, le ${ }^{222} \mathrm{Rn}$ est mesuré à Gif-sur-Yvette par l'intermédiaire de ses descendants à vie courte, les polonium-214 et -218 , et le bismuth-214, avec la méthode dite du dépôt actif. Cette méthode consiste à recueillir les descendants du radon sur un filtre et à l'analyser régulièrement pour mesurer l'activité radioactive de ces derniers. Décroissante au cours du temps, la mesure cette activité radioactive permet de remonter à la quantité initiale de radon-222.

Entre janvier 2007 et mai 2009, un analyseur de radon à double filtre a été installé à Gif-sur-Yvette avec l'assistance de l'Australian Nuclear Science and Technology Organisation (ANSTO) [Zahorowski et al., 2004]. Depuis mai 2009 , cet analyseur mesure le ${ }^{222} \mathrm{Rn}$ sur le site de Trainou à $180 \mathrm{~m}$ de hauteur. Avec cette méthode, le radon-222 émet un rayonnement alpha en se désintégrant. Ce dernier est converti en photons qui sont comptés par un photomultiplicateur. En mesurant un échantillon de référence, on peut donc calculer la concentration de radon-222. Un second analyseur utilisant la méthode du dépôt actif a été installé, en mai 2010, à $50 \mathrm{~m}$ de hauteur.

\section{La méthode Radon}

Pour étudier le dépôt de $\mathrm{H}_{2}$ aux échelles locale et régionale, nous avons utilisé la méthode dite Radon à Gif-surYvette et à Trainou, pour les deux niveaux instrumentés, avec un analyseur radon.

Sur la figure 2, les concentrations de $\mathrm{H}_{2}$, CO et ${ }^{222} \mathrm{Rn}$ sont tracées pour plusieurs jours de septembre 2007. Pendant la journée, les concentrations mesurées représentent le mélange des flux, de dépôt et d'émissions, principalement celles dues aux combustibles fossiles, c'est-à-dire liées au transport. Pendant la nuit, lorsque les émissions dues au transport sont négligeables, on voit clairement une corrélation entre l'augmentation de la concentration de ${ }^{222} \mathrm{Rn}$ et la diminution de celle de $\mathrm{H}_{2}$. $\mathrm{CO}$ nous sert à vérifier que la diminution de concentration de $\mathrm{H}_{2}$ est bien due au dépôt et non à une diminution progressive des émissions communes de $\mathrm{H}_{2}$ et $\mathrm{CO}$. Pour les nuits présentées sur la figure 2, la concentration de $\mathrm{CO}$ reste stable.

La méthode Radon a été appliquée aux observations nocturnes de $\mathrm{H}_{2}$ (23 h$4 \mathrm{~h}$ ) afin de privilégier les événements où le dépôt domine les autres termes. À Gif-sur-Yvette et à Trainou, les émissions anthropiques, durant cette période, représentent moins de $5 \%$ du dépôt de $\mathrm{H}_{2}$ et sont donc négligées. 


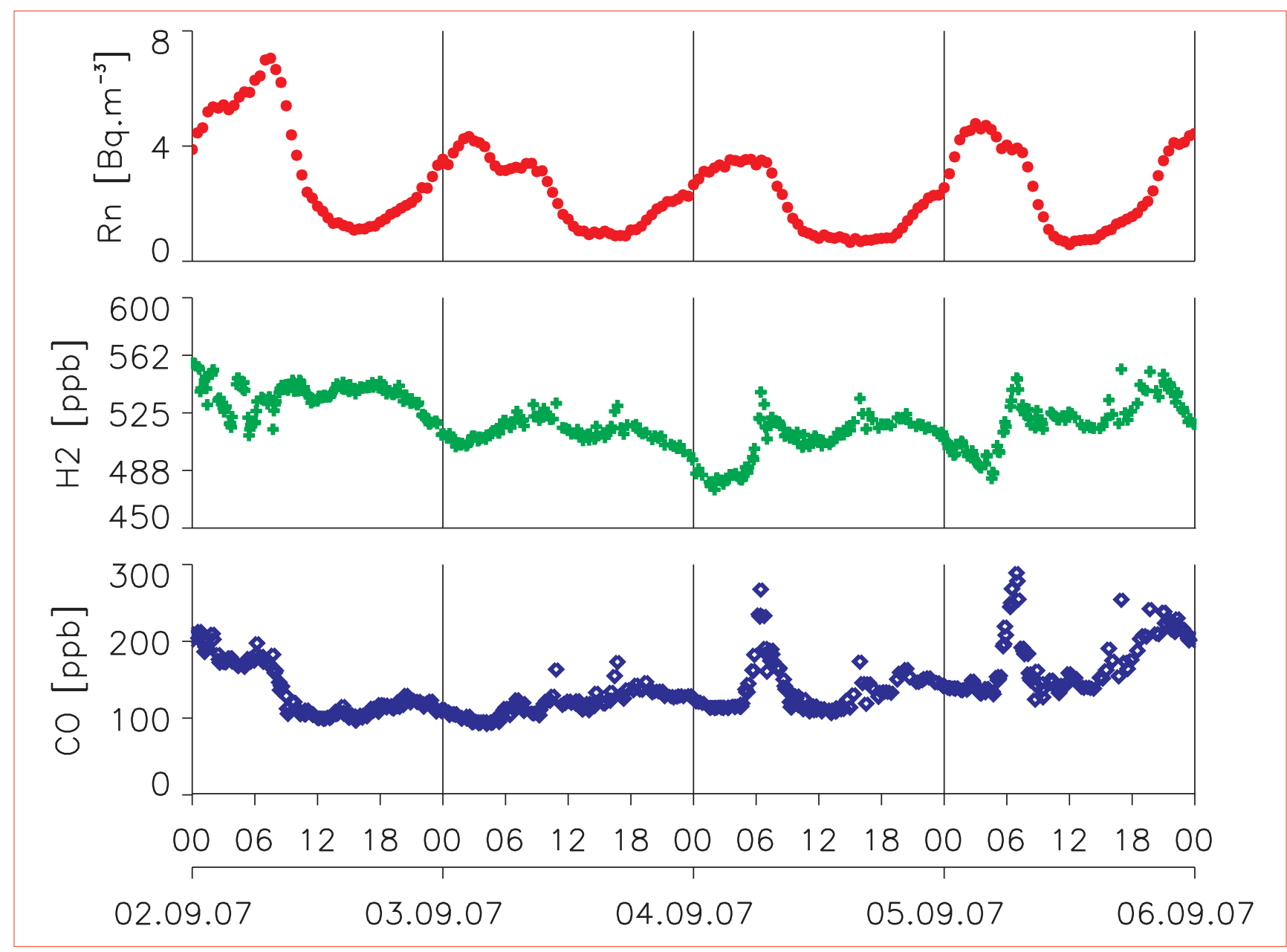

Figure 2 - Concentrations en 222Rn, $\mathrm{H}_{2}$ et $\mathrm{CO}$ à Gif-sur-Yvette, pendant quatre jours, en septembre 2007. Quatre anticorrélations nocturnes entre $\mathrm{H}_{2}$ et 222Rn sont visibles. CO ne suit pas ce même schéma. L'axe des abscisses indique la date comme jour.mois.an avec, au-dessus, les heures UTC.

\section{Résultats}

Les résultats de la méthode Radon sont présentés sur la figure 3. La majorité des événements sélectionnés se situe en été et en automne. Pendant l'hiver et le printemps, le dépôt est

Figure 3 - Vitesse de dépôt de $\mathrm{H}_{2}$ à Gif-sur-Yvette et à Trainou, à 50 et à 180 m, de janvier 2007 à septembre 2010

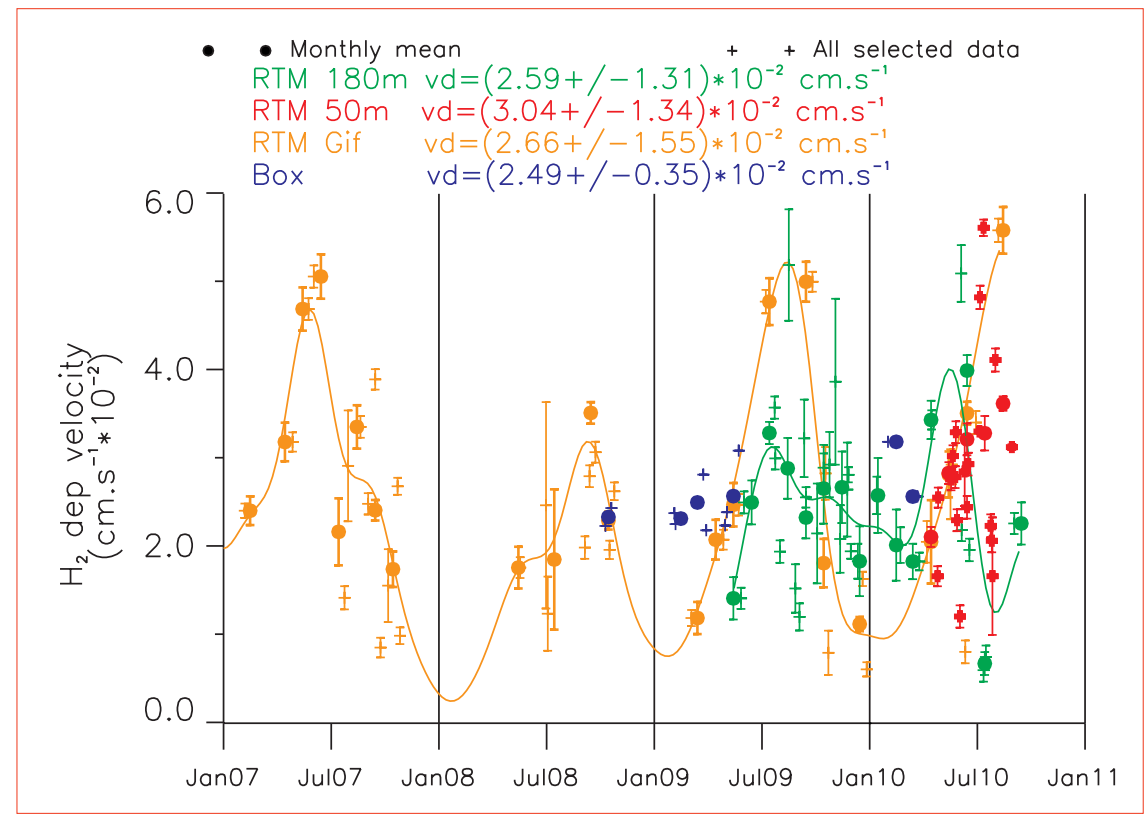

soit nul soit trop faible pour être mesuré par cette méthode. Au total, à Gif-sur-Yvette comme à Trainou, entre 2007 et 2011, seulement 5 à $7 \%$ des nuits sont sélectionnées par la méthode Radon.

À Gif-sur-Yvette, j'ai calculé une vitesse de dépôt moyenne de $2,6 \pm 1,510^{-2} \mathrm{~cm} \mathrm{~s}^{-1}$ avec une amplitude saisonnière de $40 \%$ (maximum en automne et minimum au printemps). La zone de validité de cette valeur de dépôt nocturne a un rayon estimé d'environ $60 \mathrm{~km}$.

À Trainou, à $180 \mathrm{~m}$, j'ai utilisé la méthode Radon ainsi qu'un modèle simple à une boîte pour estimer le dépôt de $\mathrm{H}_{2}$. La vitesse de dépôt moyenne est remarquablement semblable à celle de Gif-surYvette pour les deux méthodes $\left(2,8 \pm 1,310^{-2} \mathrm{~cm} \mathrm{~s}^{-1}\right)$. Le cycle saisonnier est également similaire à celui de Gif-sur-Yvette en termes de temporalité mais son amplitude est environ deux fois plus petite.

Enfin, à Trainou, la vitesse de dépôt calculée à $50 \mathrm{~m}$, pour les mois de mai et juin 2010, vaut $4,1 \pm 2,010^{-2} \mathrm{~cm} \mathrm{~s}^{-1}$ et correspond aux valeurs estivales de Gif-sur-Yvette et de Trainou à $180 \mathrm{~m}$. Pour les mesures à cette hauteur, le rayon de la zone de validité est estimé en moyenne à $90 \mathrm{~km}$. 


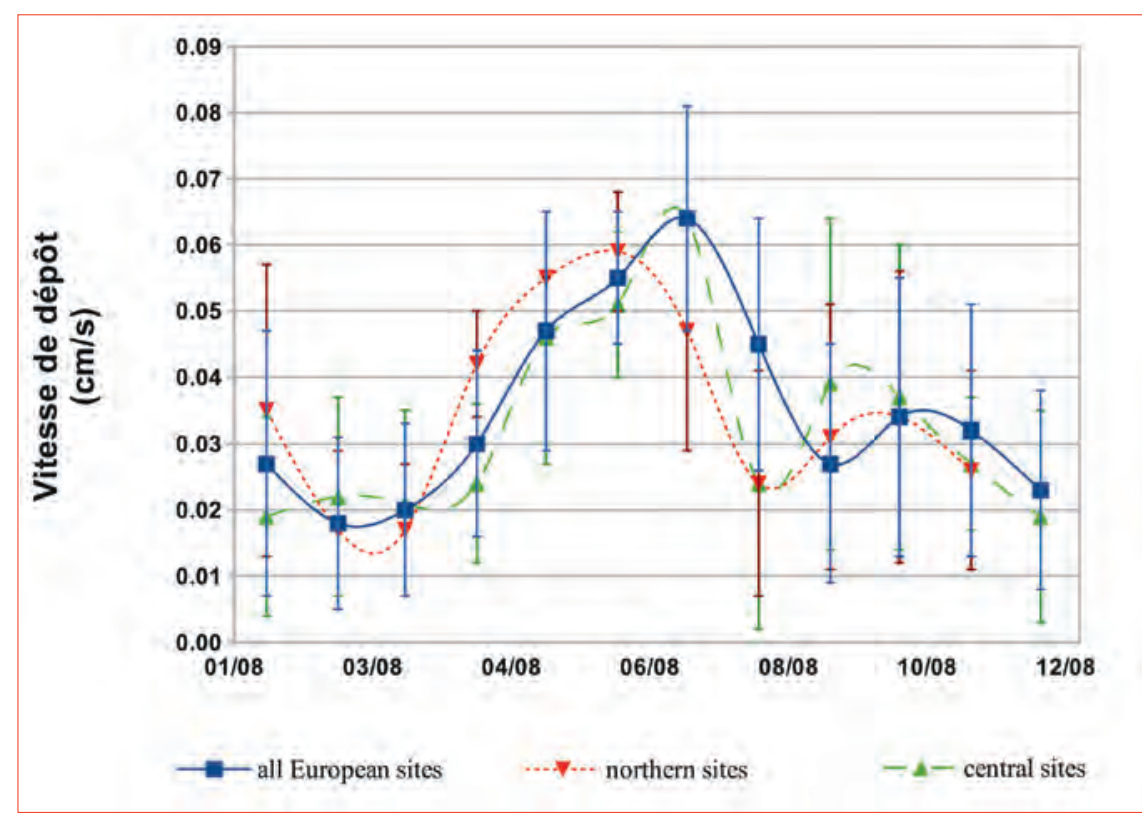

Figure 4 - Vitesses de dépôt de $\mathrm{H}_{2}$ en Europe, mesurées à l'aide de chambres de flux.

Ces résultats peuvent être comparés aux mesures ponctuelles effectuées avec des chambres de flux, en Europe, et regroupées par Schillert (2010). La figure 4 présente un récapitulatif du cycle annuel pour l'ensemble de l'Europe, pour l'Europe centrale et pour l'Europe du Nord. Le maximum de la vitesse de dépôt du $\mathrm{H}_{2}$ se situe, en moyenne, plus tôt que pour nos sites de mesures, soit vers la mi-juillet. En revanche, le minimum est en bon accord avec celui déterminé par nos mesures. La valeur moyenne pour l'ensemble de l'Europe est de 3,5 $\pm 1,510^{-2} \mathrm{~cm} \mathrm{~s}^{-1}$, avec un maximum de $6,510^{-2} \mathrm{~cm} \mathrm{~s}^{-1}$ et un minimum de $1,910^{-2} \mathrm{~cm} \mathrm{~s}^{-1}$, ce qui est en bon accord avec la méthode Radon, compte tenu des incertitudes. Le dépôt de $\mathrm{H}_{2}$ est donc relativement homogène en Europe.

\section{Passer de l'échelle locale à l'échelle globale}

\section{La modélisation inverse : principe et composantes}

La vitesse de dépôt à Gif-sur-Yvette a été intégrée avec les mesures de vitesse de dépôt réalisées au sein du projet européen EUROHYDROS(1) (Engel et al., 2009) pour établir une carte globale du dépôt de $\mathrm{H}_{2}$ (voir figure 5 , en bas). Deux autres cartes du dépôt de $\mathrm{H}_{2}$ provenant d'autres études (Hauglustaine et Ehhalt, 2002 ; Sitch et al., 2003) ont également été utilisées. Ces cartes, ainsi que d'autres contenant des informations sur les émissions de $\mathrm{H}_{2}$, ont été utilisées comme estimation de départ (a priori) pour la modélisation inverse. En effet, pour l'étude de phénomènes à grande échelle, la modélisation est l'outil idéal. Pour l'étude du dépôt, nous avons choisi la modélisation inverse. C'est un outil qui permet d'optimiser les flux à

(1) EUROHYDROS : EUROpean network for atmospheric HYDRogen Observations and Studies.

$\nabla$ Figure 5 - Cartes de vitesse de dépôt de $\mathrm{H}_{2}$ utilisées pour la modélisation (échelle de couleur : vitesse de dépôt $\times 1000(\mathrm{~cm} / \mathrm{s}))$. En haut, la carte tirée de Hauglustaine et Ehhalt (2002) ; au milieu, celle tirée de Sitch et al. (2003) ; et, en bas, celle calculée par extrapolation des mesures européennes (Søvde et al., 2008 ; Schillert, 2010)

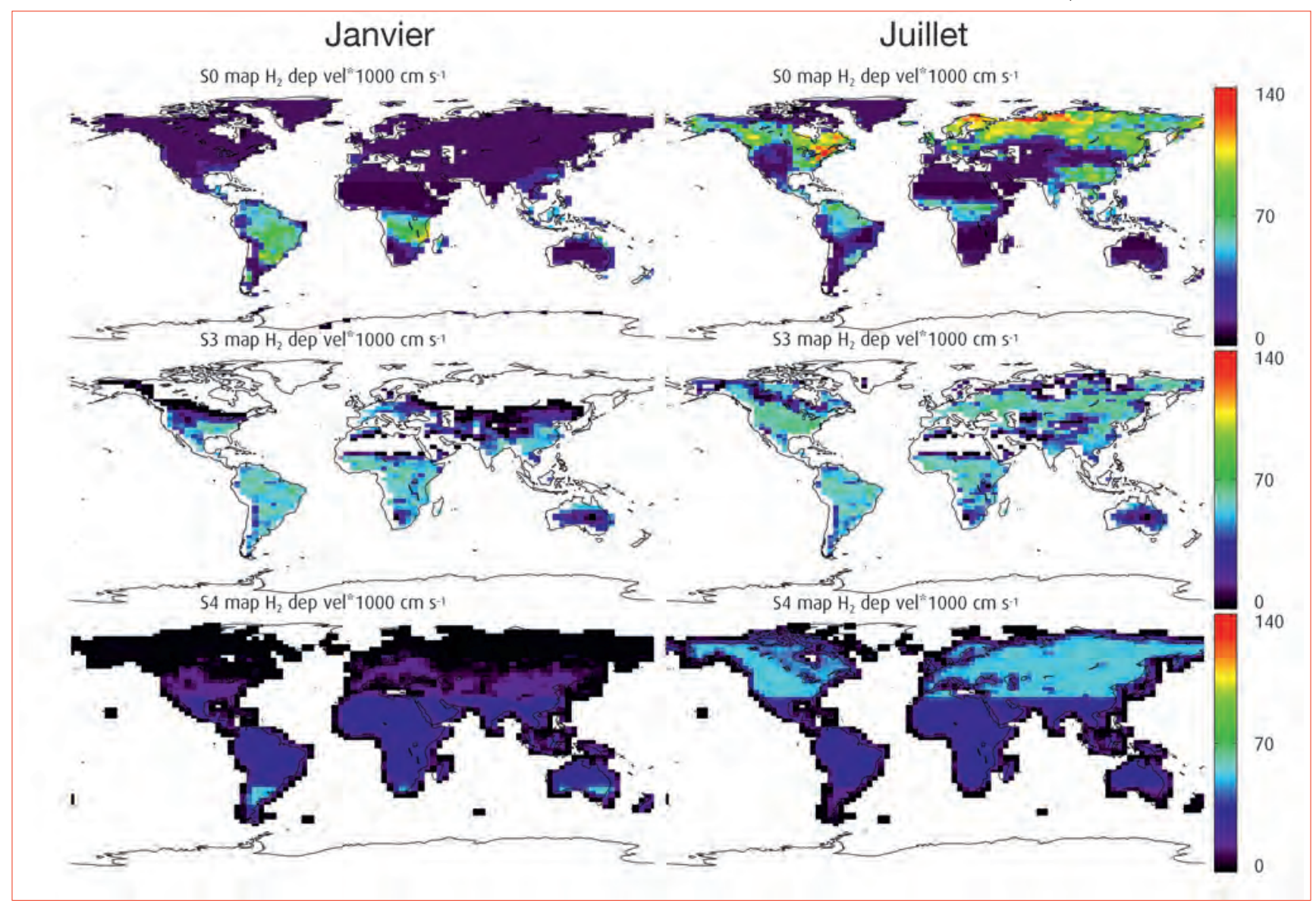


l'origine des mesures en combinant de façon optimale un modèle et des mesures. Pour cela, le modèle cherche à minimiser la différence entre les mesures et les concentrations simulées tout en tenant compte des valeurs attribuées a priori aux flux que l'on cherche à optimiser. Nous avons utilisé le modèle de chimie-transport LMDz-SACS(1) (Hourdin et Talagrand, 2006) intégré au système d'inversion PYVAR(2) (Chevallier et al., 2005).

Plusieurs scénarios ont été testés. Dans les deux premiers, le flux total de $\mathrm{H}_{2}$ (sources - puits) est inversé et la répartition entre chaque terme du bilan se fait à la fin de la simulation en utilisant les proportions de l'a priori. La carte de dépôt utilisée provient du travail de Hauglustaine et Ehhalt (2002) [voir figure 5 , en haut]. Dans le deuxième scénario, la valeur du dépôt de $\mathrm{H}_{2}$ a été augmentée afin d'obtenir des concentrations simulées plus réalistes. Pour les trois scénarios suivants, le dépôt de $\mathrm{H}_{2}$ est inversé séparément des autres termes et les trois différentes cartes de dépôt ont été testées. L'augmentation du dépôt ajoutée pour le deuxième scénario est conservée.

Pour toutes les simulations, les mesures des concentrations de $\mathrm{H}_{2}$ proviennent des stations de mesure du réseau EUROHYDROS ainsi que de celles du réseau RAMCES(3) (cf. figure 6).

\section{Résultats}

Ces inversions de $\mathrm{H}_{2}$ sont les premières à utiliser des données atmosphériques récentes (2006-2009) et continues, pour une partie des sites de mesures, ainsi qu'une résolution à l'échelle du point de grille et non par bande de latitude ou par grandes régions.

Sur la figure 7 sont représentées les concentrations observées et simulées avant inversion (à gauche) et après inversion (à droite) pour quatre stations du réseau RAMCES. Les concentrations simulées par le premier scénario, en rouge, présentent, avant inversion, un biais d'environ $100 \mu \mathrm{mol} \mathrm{mol}{ }^{-1}$. Dans les deux cas suivants, en violet (superposé au jaune), le dépôt de $\mathrm{H}_{2}$ a été augmenté et le biais a été supprimé. Après

(1) LMDz-SACS : Laboratoire de Météorologie Dynamique general circulation model Zoom Simplified Atmospheric Chemistry System. (2) PYVAR : système d'assimilation variationnelle pour gaz à effet de serre et aérosols.

(3) RAMCES : Réseau atmosphérique de mesure des composés à effet de serre.

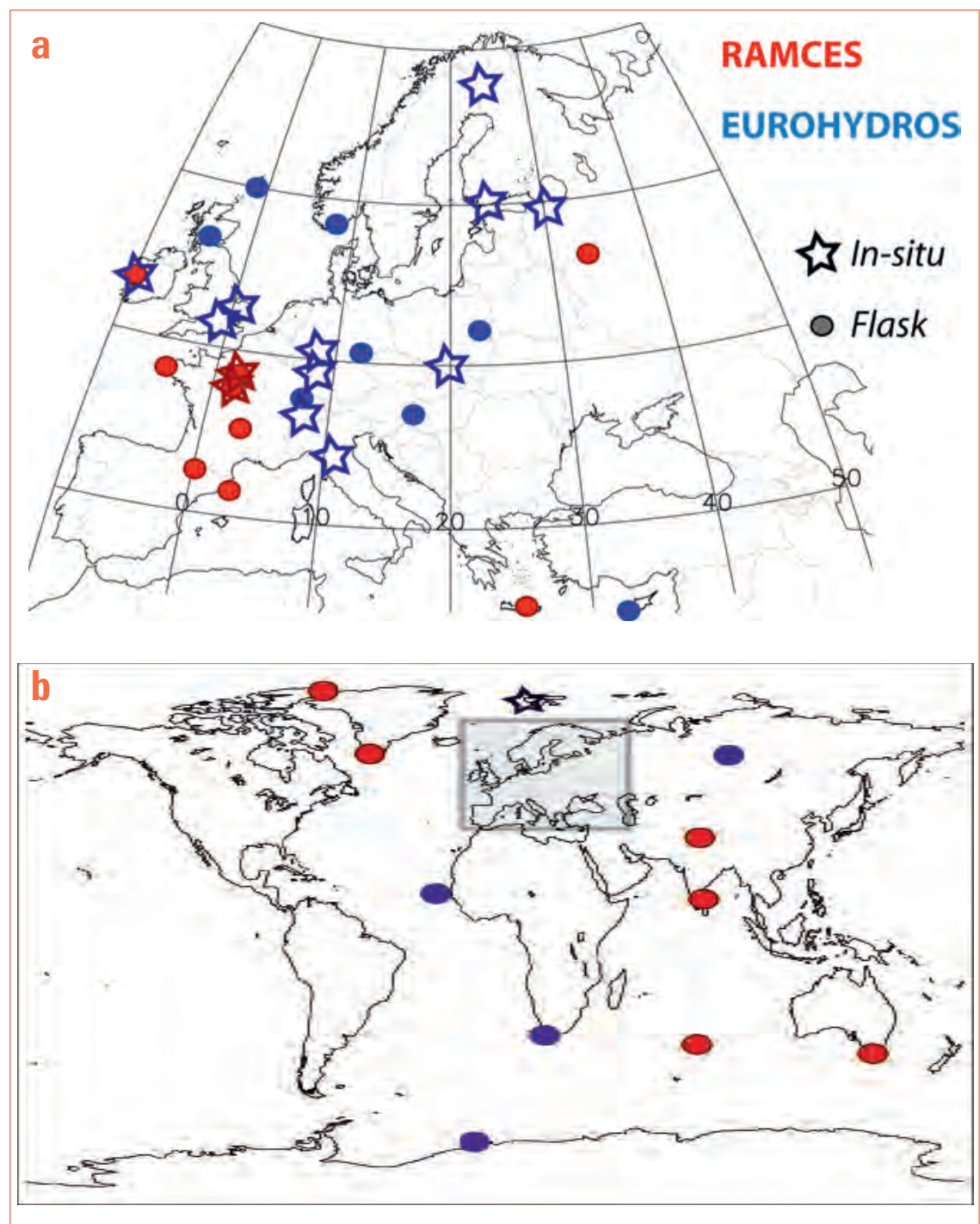

Figure 6 - Localisation des stations des réseaux de mesure EUROHYDROS et RAMCES. inversion, comme attendu, les concentrations simulées sont très proches des observations, quel que soit le scénario.
Les flux ont donc été optimisés. Sur la figure 8 , le cycle saisonnier moyen du dépôt de $\mathrm{H}_{2}$, calculé pour les trois
Figure 7 - Concentration de $\mathrm{H}_{2}$ mesurée et simulée, à gauche, avant inversion, à droite, après inversion pour quatre stations de mesures (latitude entre parenthèses). Les mesures sont en noir et les concentrations simulées en couleurs.

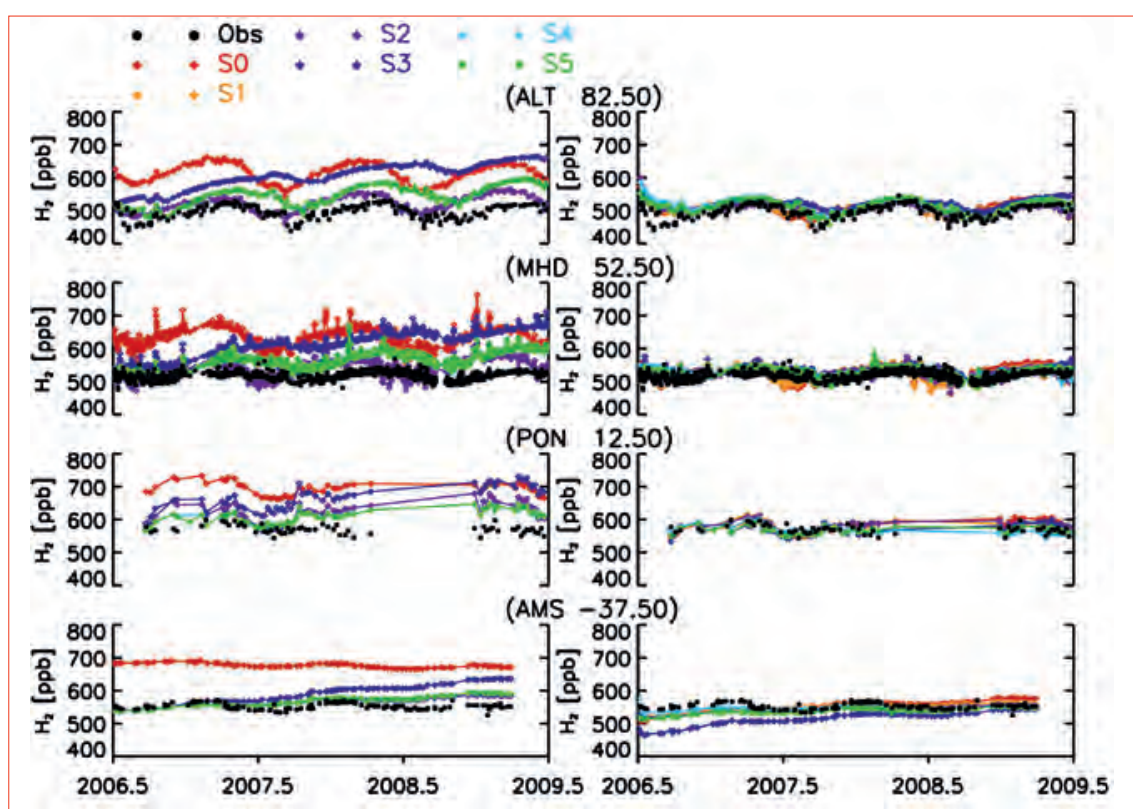




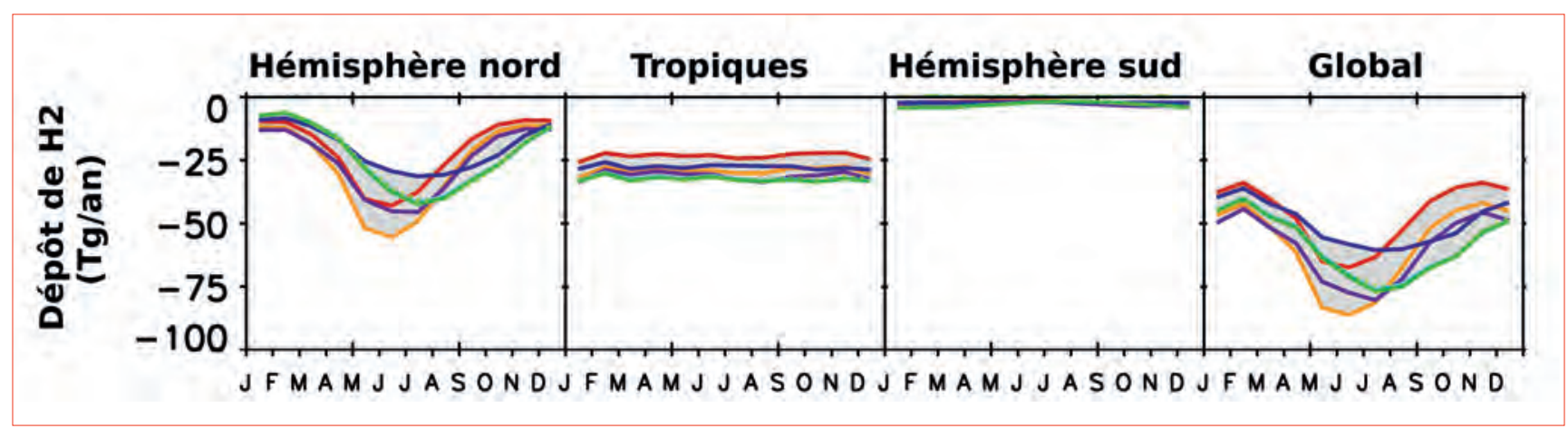

Figure 8 - Cycle saisonnier de la vitesse de dépôt de $\mathrm{H}_{2}$ simulée pour trois bandes de latitude (hémisphère nord, tropiques et hémisphère sud).

années de simulation, est représenté pour l'hémisphère nord, pour les tropiques et pour l'hémisphère sud. Dans l'hémisphère sud, le dépôt de $\mathrm{H}_{2}$ est presque nul étant donnée la faible superficie de terres émergées. Dans les tropiques, quel que soit le scénario, le dépôt reste constant au cours du temps et il est d'environ -30 Tg/an. Dans l'hémisphère nord, au contraire, on observe un fort cycle saisonnier, avec le minimum de dépôt en hiver et le maximum en été. L'augmentation du dépôt pour le deuxième scénario (en jaune) est clairement visible en été. Après inversion (scénarios 2, 3 et 4), le dépôt est plus proche de celui du premier scénario, sans augmentation. L'hypothèse d'un dépôt trop faible, entraînant des concentrations simulées non réalistes, est donc invalidée par le modèle. De plus, on observe un décalage du maximum du dépôt du mois de juin au mois d'août, ce qui correspond aux flux calculés (cf. figures 3 et 4).

Le meilleur scénario en termes de saisonnalité dans l'hémisphère nord est celui qui utilise une carte de vitesse de dépôt de $\mathrm{H}_{2}$ élaborée à partir de mesures de ce dépôt. Avec ce scénario, le puits global de $\mathrm{H}_{2}$ est estimé à $-59 \pm 9 \mathrm{Tg} \mathrm{an}^{-1}$. Il se situe à $95 \%$ audessus de $30^{\circ} \mathrm{S}$, dont $38 \%$ au-dessus de $30^{\circ} \mathrm{N}$ et $57 \%$ dans les régions tropicales. L'incertitude indiquée cidessus correspond à la variation entre les différents scénarios et représente donc l'incertitude minimum.

\section{Conclusion}

Les processus physiques et chimiques du dépôt de dihydrogène dans les sols ne sont encore connus qu'avec de grandes incertitudes. Toutefois l'utilisation de mesures atmosphériques et la modélisation nous ont permis de mieux estimer ce dépôt en déterminant son importance localement mais aussi globalement. Les études menées à Gif-sur-Yvette et à Trainou indiquent que la méthode Radon est fiable pour estimer les flux de $\mathrm{H}_{2}$ à l'échelle locale. De plus, les résultats pointent vers des valeurs de vitesse de dépôt relativement homogènes en Europe. La valeur globale du dépôt de $\mathrm{H}_{2}$, estimée par inversion, est en accord avec les études publiées précédemment et résumées, pour la plupart, par Ehhalt et Rohrer (2009).

La plupart des nouvelles études utilisent déjà la modélisation pour exprimer les flux de $\mathrm{H}_{2}$ mais l'utilisation de l'inversion et la prise en compte des mesures d'un réseau étendu de stations est plus rare. Cependant, depuis 2009, la concentration du dihydrogène doit être calculée en utilisant la même valeur de référence, quel que soit le laboratoire. Ainsi peut-on imaginer, dans un avenir proche, une nouvelle simulation qui regrouperait les mesures de concentration du $\mathrm{H}_{2}$ de l'ensemble des stations dans le monde avec la même valeur de référence. À la trentaine de stations gérées par les participants du projet EUROHYDROS pourraient s'ajouter une cinquantaine de stations, situées essentiellement aux États-Unis et gérées par le ESRL/NOAA(1). Sans nul doute cette nouvelle étude permettraitelle de réduire encore, et de façon importante, l'incertitude sur les flux globaux de dihydrogène.

\section{Remerciements}

Un grand merci à Philippe Bousquet, à Martina Schmidt et à Michel Ramonet qui m'ont accompagnée pendant mes années de thèse. Merci également aux membres du jury qui m'ont attribué le prix André Prud'homme 2011.

(1) ESRL/NOAA : Earth System Research Laboratory/National Oceanic and Atmospheric Administration

\section{Bibliographie}

\footnotetext{
: Chevallier F., M. Fisher, P. Peylin, S. Serrar, P. Bousquet, F. Bréon, A. Chédin et P. Ciais, 2005 : Inferring CO2 sources and sinks from satellite observations: Method - and application to TOVS data. Journal of Atmospheric Chemistry, 110, D24309.

- Conrad R. et W. Seiler, 1981 : Decomposition of atmospheric hydrogen by soil microorganisms and soil enzymes. Soil Biology and Biochemistry, 13(1), 43-49.

Conrad R. et W. Seiler, 1985 : Influence of temperature, moisture, and organic carbon on the flux of $\mathrm{H}_{2}$ and $\mathrm{CO}$ between soil and atmosphere: Field studies in subtropical regions. J. Geophys. Res., 90, 5699-5709.

Constant P., L. Poissant et R. Villemur, 2008 : Isolation of Streptomyces sp. PCB7, the first microorganism demonstrating high-affinity uptake of tropospheric $\mathrm{H}_{2}$. The ISME Journal, 2, 1066-1076.

Ehhalt D. H. et F. Rohrer, 2009 : The tropospheric cycle of $\mathrm{H}_{2}$ : A critical review. Tellus B, 61, 500-535.

- Engel A. et al., 2009 : EUROHYDROS, a European Network for atmospheric hydrogen observations and studies. EUROHYDROS Final Report.
} 
- Guo R. et R. Conrad, 2008 : Extraction and characterization of soil hydrogenases oxidizing atmospheric hydrogen. Soil Biology and Biochemistry, 40(5), $1149-1154$.

- Hauglustaine D. A. et D. H. Ehhalt, 2002 : A three-dimensional model of molecular hydrogen in the troposphere. J. Geophys. Res., 107, 4330, doi:10.1029/2001JD001156.

- Hourdin F. et O. Talagrand, 2006 : Eulerian backtracking of atmospheric tracers. I: Adjoint derivation and parametrization of subgrid-scale transport. Ouart. J. Roy. Meteorol. Soc., 132, 567-583.

- Levy H., 1971 : Normal atmosphere: Large radical and formaldehyde concentrations predicted. Science, 173, 141-143.

Schery S. D., D. H. Gaeddert et M. H. Wilkening, 1984 : Factors affecting exhalation of radon from a gravelly sandy loam. J. Geophys. Res., 89, 7299-7309.

- Schillert A., 2010 : Parameters influencing the seasonality of $\mathrm{H}_{2}$ uptake in soils. Thèse.

Schmidt U., 1974 : Molecular hydrogen in the atmosphere. Tellus, 26, 78-90.

Sitch S., B. Smith, I. C. Prentice, A. Arneth, A. Bondeau, W. Cramer, J. O. Kaplan, S. Levis, W. Lucht, M. T. Sykes, K. Thonicke et S. Venevsky, 2003 - Evaluation of ecosystem dynamics, plant geography and terrestrial carbon cycling in the LPJ dynamic global vegetation model. Global Change Biology, 9(2), 161-185.

- Smith-Downey N. V., J. T. Randerson et J. M. Eiler, 2006 : Temperature and moisture dependence of soil H2 uptake measured in the laboratory. Geophys. Res. Lett., - 33, L14813.

- Søvde O. A., M. Gauss, S. P. Smyshlyaev et I. S. A. Isaksen, 2008 : Evaluation of the chemical transport model 0slo CTM2 with focus on arctic winter ozone depletion. J. Geophys. Res., 113, D09304, doi:10.1029/2007JD009240.

- Yonemura S., S. Kawashima et H. Tsuruta, 1999 : Continuous measurements of CO and H2 deposition velocities onto an andisol: Uptake control by soil moisture. Tellus B, 51, 688-700.

- Yonemura S., S. Kawashima et H. Tsuruta, 2000a : Carbon monoxide, hydrogen, and methane uptake by soils in a temperate arable field and a forest. J. Geophys. Res. 105, 14347-14362.

Yonemura S., M. Yokozawa, S. Kawashima et H. Tsuruta, 2000b : Model analysis of the influence of gas diffusivity in soil on CO and $\mathrm{H}_{2}$ uptake. Tellus B, 52 , 919-933.

- Yver C., M. Schmidt, P. Bousquet, W. Zahorowski et M. Ramonet, 2009 : Estimation of the molecular hydrogen soil uptake and traffic emissions at a suburban site - near Paris through hydrogen, carbon monoxide, and radon-222 semicontinuous measurements. J. Geophys. Res., 114, D18304, doi:10.1029/2009JD012122.

: Yver C., M. Schmidt, P. Bousquet et M. Ramonet, 2011a : Measurements of molecular hydrogen and carbon monoxide on the Trainou tall tower. Tellus B, 63, 52-63.

- Yver C., I. C. Pison, A. Fortems-Cheiney, M. Schmidt, F. Chevallier, M. Ramonet, A. Jordan, O. A. Søovde, A. Engel, R. E. Fisher, D. Lowry, E. G. Nisbet, I. - Levin, S. Hammer, J. Necki, J. Bartyzel, S. Reimann, M. K. Vollmer, M. Steinbacher, T. Aalto, M. Maione, J. Arduini, S. O’Doherty, A. Grant, W. T. Sturges, - G. L. Forster, C. R. Lunder, V. Privalov, N. Paramonova, A. Werner et P. Bousquet, 2011b : A new estimation of the recent tropospheric molecular hydrogen budget - using atmospheric observations and variational inversion. Atm. Chem. Phys., 11, 3375-3392.

Zahorowski W., S. D. Chambers et A. Henderson-Sellers, 2004 : Ground based radon-222 observations and their application to atmospheric studies. Journal of : Environmental Radioactivity, 76, 3-33. 